\title{
Discrimination of face gender and expression under dual-task conditions
}

\author{
Ana García-Gutiérrez ${ }^{1}$ - Luis Aguado ${ }^{1}$ - Verónica Romero-Ferreiro ${ }^{1}$. \\ Elisa Pérez-Moreno ${ }^{1}$
}

Published online: 22 November 2016

(C) The Psychonomic Society, Inc. 2016

\begin{abstract}
In order to test whether expression and gender can be attended to simultaneously without a cost in accuracy four experiments were carried out using a dual gender-expression task with male and female faces showing different emotional expressions that were backward masked by emotionally neutral faces. In the dual-facial condition the participants had to report both the gender and the expression of the targets. In two control conditions the participant reported either the gender or the expression of the face and indicated whether a surrounding frame was continuous or discontinuous. In Experiments 1-3, with angry and happy targets, asymmetric interference was observed. Gender discrimination, but no expression discrimination, was impaired in the dual-facial condition compared to the corresponding control. This effect was obtained with a between-subjects design in Experiment 1, with a within-subjects design in Experiment 2, and with androgynous face masks in Experiment 3. In Experiments $4 \mathrm{a}$ and $4 \mathrm{~b}$ different target combinations were tested. No decrement of performance in the dual-facial task was observed for either gender or expression discrimination with fearful-disgusted (Experiment 4a) or fearfulhappy faces (Experiment 4b). We conclude that the ability to attend simultaneously to gender and expression cues without a decrement in performance depends on the specific combination of expressions to be differentiated between. Happy and angry expressions are usually directed at the perceiver and command preferential attention. Under conditions of restricted viewing such as those of the present study, discrimination of these expressions is prioritized leading to impaired discrimination of other facial properties such as gender.
\end{abstract}

Ana García-Gutiérrez

anagarci@ucm.es

1 Facultad de Psicología, Universidad Complutense, Campus de Somosaguas, 28223 Madrid, Spain
Keywords Face recognition · Face perception · Dual-task performance

\section{Introduction}

Selective attention is needed for visual processing due to the limited capacity of the attentional system to process perceptual information (see Desimone \& Duncan, 1995; Itti \& Koch, 2001; Marois \& Ivanoff, 2005, for reviews and relevant theory). One important property influencing selective attention is the emotional or affective value of the stimulus. There is abundant behavioral and neuroscientific evidence that the affective significance or "emotional salience" of sensory events exerts a strong influence on attention, facilitating stimulus processing and drawing attention to emotionally relevant stimuli, sometimes at the expense of other information that might be simultaneously present (see reviews by Compton, 2003; Schupp, Flaisch, Stockburger, \& Junghöfer, 2006; Vuilleumier, 2005).

Faces showing emotional expressions are a natural class of affective stimuli for human beings. Studies employing different methodologies converge in showing that facial expressions of emotion possess a strong power to capture attention and are processed and identified with high efficiency. First, detection of emotional faces is especially efficient as shown by its lower perceptual threshold (Calvo \& Esteves, 2005), accurate identification with brief exposure times (e.g., Calvo \& Lundqvist, 2008; Roesch et al., 2010), and resistance to backward masking (e.g., Aguado, Serrano-Pedraza, \& García-Gutiérez, 2014; Maxwell \& Davidson, 2004; Milders, Sahraie, \& Logan, 2008). Second, an attentional bias has been found for the detection of emotional faces in complex stimulus arrays. Visual search studies where emotional faces are presented among distracters have shown efficient detection of emotional targets embedded in complex stimulus arrays (e.g., Juth, Lundqvist, Karlsson, \& Öhman, 
2005; Öhman, Lundqvist, \& Esteves, 2001; Pinkham et al., 2010; see the review by Frischen, Eastwood, \& Smilek, 2008). Finally, neuroscientific studies have shown that modulation of brain activity by emotional expressions occurs at early processing stages and can be obtained even when the faces appear outside of the focus of attention or when perception is impaired by backward masking (e.g., Batty \& Taylor, 2003; Morris, Öhman, \& Dolan, 1998; Mogg \& Bradley, 1999; Vuillemier et al., 2001). All these results converge in showing that emotional expressions are especially salient stimuli that are processed in a fast and efficient fashion, sometimes at the expense of other information that may be simultaneously present (see also Pérez-Moreno, Romero-Ferreiro \& García-Gutiérrez, 2016).

Theories of face perception (Bruce \& Young, 1986; Haxby, Hoffman, \& Gobbini, 2000) and empirical evidence indicate that different face-processing tasks such as identifying the expression or the gender of a face are based on the use of at least partially different types or amounts of visual information (Atkinson, Tipples, Burt, \& Young, 2005; Schyns \& Oliva, 1999; Schweinberger \& Soukoup, 1998) and rely on the activity of different neural systems (Dzhelyova, Ellison, \& Atkinson, 2011; Haxby et al., 2000; Mouchetant-Rostaing, Giard, Bentin, Aguera, \& Pernier, 2000). For example, the traditional Bruce and Young model of face perception (1986) proposes a distinction between a structural code that underlies face recognition through the processing of fixed aspects of faces and an expression code, which underlies recognition of the dynamic changes of facial features that compose facial expressions of emotion. However, the theoretically relevant issue of the extent to which processing of different facial properties can run in parallel has not been considered before. For example, Haxby et al.'s brain systems model (2000) proposes two parallel, separate systems for the analysis of invariant (identity, gender) and changeable (expression, eye gaze) facial properties. According to this, one would expect that gender and expression could be processed and attended concurrently. However, the limited nature of visual attention and the high saliency of emotional expressions might restrict our ability to attend simultaneously to these two facial properties and give primacy to expression processing. In the present study we ask whether the gender and expression of faces can be accurately identified from briefly presented faces when the demands of a dual task require simultaneous attention to both. Exploring this possibility might provide evidence relevant for the more general issues of the primacy of expression processing in face perception, the relationship between the processing of different facial properties, and the distribution of attentional resources in face categorization tasks.

There are no parallel results to those obtained with emotional expressions in visual search or backward masking studies that suggest a superior attention-grabbing power of face gender. However, studies using other experimental paradigms have shown fast and efficient identification of this facial property. Accurate gender identification has been reported with short exposure times over $50 \mathrm{~ms}$ in a single task paradigm (Roesch et al., 2010). Moreover, similarly fast and efficient extraction of gender and emotion information from complex stimulus arrays has also been shown (Haberman \& Whitney, 2007). However, other studies have provided results that suggest slower decoding of gender than expression. For example, optimal gender identification seems to require slightly longer exposure times (Aguado et al., 2014). On the other hand, studies using the event-related potentials (ERP) technique have revealed that early potentials linked to visual processing of faces are modulated by the expression but not by the gender of faces that has an effect on later ERP components (e.g., Mouchetant-Rostaing et al., 2000; Mouchetant-Rostaing \& Giard, 2003; Valdés-Conroy et al., 2014). Finally, nearnormal gender performance has been reported by Reddy, Wilken, and Koch (2004) in a dual-task paradigm that combined gender categorization with a letter-search task. Although this last result shows that gender categorization can resist the demands of a dual task, a different matter is if it could also be performed with no cost in accuracy when the gender and expression have to be attended simultaneously.

The present paper reports the results of experiments aimed at studying expression and gender categorization under dual-task conditions. Participants were unpredictably asked after each trial to report either of these facial properties. Moreover, in order to tax visual attention and increase the probability of finding interference effects, the target faces were presented with short exposure times. Finally, precise control of exposure time was achieved by presenting immediately after termination of the target face a different, expressively neutral face that acted as a backward mask. The expressions employed as targets were those corresponding to the emotions of happiness and anger that are typically used in studies showing facilitated visual search for emotional faces. In this type of study happy-superiority (Becker et al., 2011; Calvo \& Nummenmaa, 2008; Craig, Becker, \& Lipp, 2014; Juth et al., 2005; Leppänen \& Hietanen, 2004) and angry-superiority effects (e.g., Fox \& Damjanovic, 2006; Horstmann \& Bauland, 2006; Pinkham et al., 2010; Shasteen, Sasson, \& Pinkhanm, 2014) have been described and interpreted as showing preferential attentional capture by the corresponding expressions (however, see Becker et al., 2011 for a critical review and contrary evidence). Of course, results obtained with these expressions do not necessarily generalize to other emotional expressions although from an evolutionary point of view one might speculate that happy and angry faces are especially effective to capture attention because of their specific communicative function that is, respectively, to signal the observer to approach or to avoid. Moreover, a general case 
cannot be made for all emotional expressions, given the variation in the efficiency with which human observers identify different emotional expressions and the differential saliency of the distinctive features of different expressions.

In the present study, performance in a dual expression/ gender task was compared to performance in one of two control conditions, each involving the discrimination of one facial property (expression or gender) and a concurrent non-facial discrimination between a solid or dashed line-frame surrounding the target face. Thus the main experimental manipulation was the context in which gender and expression discrimination occurred. In the dual-facial task, gender discrimination was performed in the context of expression discrimination and vice versa. However, in the dual-control conditions either gender or expression categorization had to be performed in the context of the discrimination of a non-facial feature. With this design, for example, comparable expression categorization in the context of the dual-facial task and in that of the corresponding control condition (i.e., the expression/frame task) would indicate no interference by gender. On the contrary, poorer performance in the dual-facial task would be indicative of such interference. Our prediction was that expression would be differentiated with similar efficiency in both task contexts. We also predicted that under the restricted viewing conditions used in the present study, attention to facial expression would have priority and that this would be in detriment of attention to gender, thus leading to reduced gender accuracy in the dual-facial task. Thus, we expected an asymmetric interference effect from expression to gender but not from gender to expression in the dual-facial task.

The second experimental important manipulation was the duration of the target faces, which was compared within-subjects, with a range of 17-143 ms. Comparing between different target durations was important because performance in the dual task might critically depend on the amount of time allowed to extract from the target faces relevant information to perform the required discriminations. One might assume in principle that shorter exposure times should impose more strict demands on visual attention and that interference effects should be more likely with these short durations. In all the experiments in the present series the target faces were immediately followed by a second neutral face that acted as a backward mask. This was done to obtain a precise control of the exposure time to the target faces. Backward masking is assumed to act by interrupting processing of the preceding target stimulus (see Breitmeyer \& Ogmen, 2000) and so it allows precise control of the time that the visual system has to process it. We selected the range of target durations based on evidence from previous studies on backward masking of emotional faces by other researchers (Esteves \& Öhman, 1993; Maxwell \& Davidson, 2004; Pessoa, Jappee, \& Ungerleider, 2005) and our own (Aguado et al., 2014).

\section{Experiment 1}

In the present experiment, a dual facial and two dual-control conditions were compared in a between-subjects design with six different durations. Participants had to categorize male and female faces showing happy or angry expressions in terms of gender and emotional expression. The dualfacial task was the target experimental condition and required the participants to categorize the faces in terms of gender or expression on different trials. In order to force participants to explicitly attend to both facial properties, they were unpredictably asked after each trial to report the gender or the expression of the face. The control conditions were also dual tasks, but in this case one of the component tasks was facial (gender or expression) and the other was not (discriminating between a solid or dash frame around the target face). Thus, the complete design included three dualtask conditions: the dual gender-expression task, the gender-control task, and the expression-control task.

The crucial comparison was between the accuracy of gender and expression discrimination in the dual-facial and control conditions. This comparison would provide an estimation of the relative cost, if any, of having to attend simultaneously to the gender and the expression of a face. While competition between facial properties might arise in the dual facial condition, no such competition should occur in the control conditions. The comparison between the dual facial and control conditions might yield three different patterns of results corresponding to no interference, symmetrical interference, and asymmetric interference. Similar gender and expression discrimination accuracy in the experimental and control conditions would indicate no interference. Reduced accuracy in both the gender and the expression tasks in the dual facial condition would indicate symmetrical interference. Finally, impaired performance in only one of the tasks (expression or gender) in the dual facial condition would be indicative of asymmetric interference. Based on the evidence discussed in the introductory section about the efficient processing and attention-grabbing power of facial expressions of emotion, we predicted that attention would be preferentially captured by emotional expression in the dualfacial task and that asymmetric interference of gender identification would thus be observed. The target faces were presented with different exposure times $(17,34,51,85$, 119 , and $143 \mathrm{~ms}$ ). This manipulation had two goals. First, the probability of competition of gender and expression for attentional resources was increased by the use of brief exposure times. Second, comparing the effects of different target durations would give crucial information on the time range over which the reported interference effects appear. Moreover, with the aim of controlling precisely the effective exposure time to the target, a neutral face mask was presented immediately after target ending. 


\section{Method}

\section{Participants}

Participants in this experiment were 36 undergraduate Psychology students ( 26 females, $M$ age $=20.29$, range 18 29 years) from the Universidad Complutense (Madrid, Spain) who received course credit in exchange. All of them had normal or corrected-to-normal vision. Twelve participants were assigned randomly to each of the three conditions included in the design.

\section{Apparatus and stimuli}

Presentation of stimuli and registration of responses were controlled with the E-Prime 2.0 software. Stimuli were presented on a VGA 17 -in. monitor (refresh rate $59.82 \mathrm{~Hz}$ ). Participants were seated at a distance of $50 \mathrm{~cm}$ from the screen with head position controlled by means of a chin rest. Responses were registered using a five-key response box (PST Serial Response Box, 200A). Sessions were carried out individually in a dimly lit room. Two different sets of faces were used as targets or masks. The targets were 32 pictures corresponding to 16 models (eight male, eight female) showing either a happy or an angry expression, taken from the KDEF collection (Lundqvist, Flykt, \& Öhman, 1998). Eight of the angry faces and the sixteen happy faces were open-mouth expressions, with teeth visible. With the aim of reducing the saliency of the white of the teeth and preventing the participants from basing their responses exclusively on this feature, the mouth area of the faces showing open-mouth expressions was blurred using a 10-pixel Gaussian filter. Moreover, images were cut to conceal most of the hair in order to avoid possible influences of hairstyling and promote attention to facial features. Finally, images were equated in contrast energy (root mean square contrast $(\mathrm{cRMS})=0.2) .{ }^{1}$ Eight expressively neutral faces from the same collection and belonging to eight different individuals (four male, four female) were used as masks. These pictures were quantized using the average density of the original images, with a square size of $8 \times 8$ pixels. This sampling size was selected based on evidence from other studies performed in our lab (Aguado et al., 2014) that showed that this quantization level has maximum masking power. The target faces were presented inside a solid or dashed line frame, which constituted the non-facial feature in the control conditions.

\footnotetext{
${ }^{1}$ See Aguado et al. (2009) for further explanation of this procedure Appendix B.
}

\section{Procedure}

Twenty-four practice trials were given using a set of KDEF faces different to that used during the experiment proper. Target faces were presented on the center of the screen subtending $6 \times 10^{\circ}$ (horizontal $\times$ vertical) of visual angle. The faces were presented on a square gray background $\left(45.2 \mathrm{~cd} / \mathrm{m}^{2}\right)$ with a size of $512 \times 512$ pixels, subtending $13.5 \times 13.5^{\circ}$ of visual angle (see Fig. 1a). Each trial was preceded by a 500 -ms fixation mark centered on the screen. Target faces were presented with different durations (17, 34, $51,85,119$, and $143 \mathrm{~ms}$ ) that alternated randomly and were immediately followed by a 750-ms neutral face mask. The gender of the target and mask faces was combined so that the two faces were of the same or different gender on $50 \%$ of the trials. Immediately after removal of the face mask a pair of words corresponding to the two options of each component task ("Happy" and "Angry," "Male" and "Female," or "Solid" and "Dash") appeared on the center of the screen acting as a prompt to respond. Finally, a varying inter-trial interval was used ( $M=2,212 \mathrm{~ms}$, range 1,500-3,000 ms). An example of the event sequence comprising each trial is shown in Fig. 1b. Participants were instructed to respond by pressing the keys associated with the corresponding four response options: happy/angry or male/female in the dualfacial condition; happy/angry or solid/dash in the expression-control condition; and male/female or solid/dash in the gender-control condition. Different pairs of keys were assigned to each component of the dual tasks. More specifically, in each group participants had to press keys 1 or 2 (with the left hand) for one of the tasks, and 4 or 5 (with the right hand) for the other task (key assignment counterbalanced between participants). To ensure that participants understood the task and the key assignment, the experimenter remained in the experimental room during the practice trials and assisted the participant if necessary. Each condition comprised 192 trials divided into six blocks, each followed by a rest period.

\section{Results}

The correct results of the between-subjects comparison of expression and gender categorization in the dual-facial task with their respective control tasks were reported. Only responses within the 200-2,000 ms range were taken into account. As the participants had to withhold their responses until the response prompt appeared, reaction times can be considered an estimation of the time taken to react to the instruction rather than to the target itself, as participants did not know what kind of response must be emitted until options appeared on the screen and the stimulus had disappeared. Note that the sample data met sphericity assumptions for mixed ANOVA in this experiment. Scores in different groups have homogeneous 
a

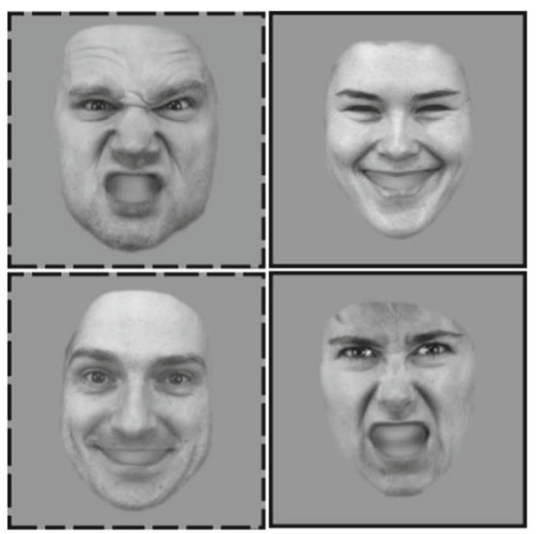

b

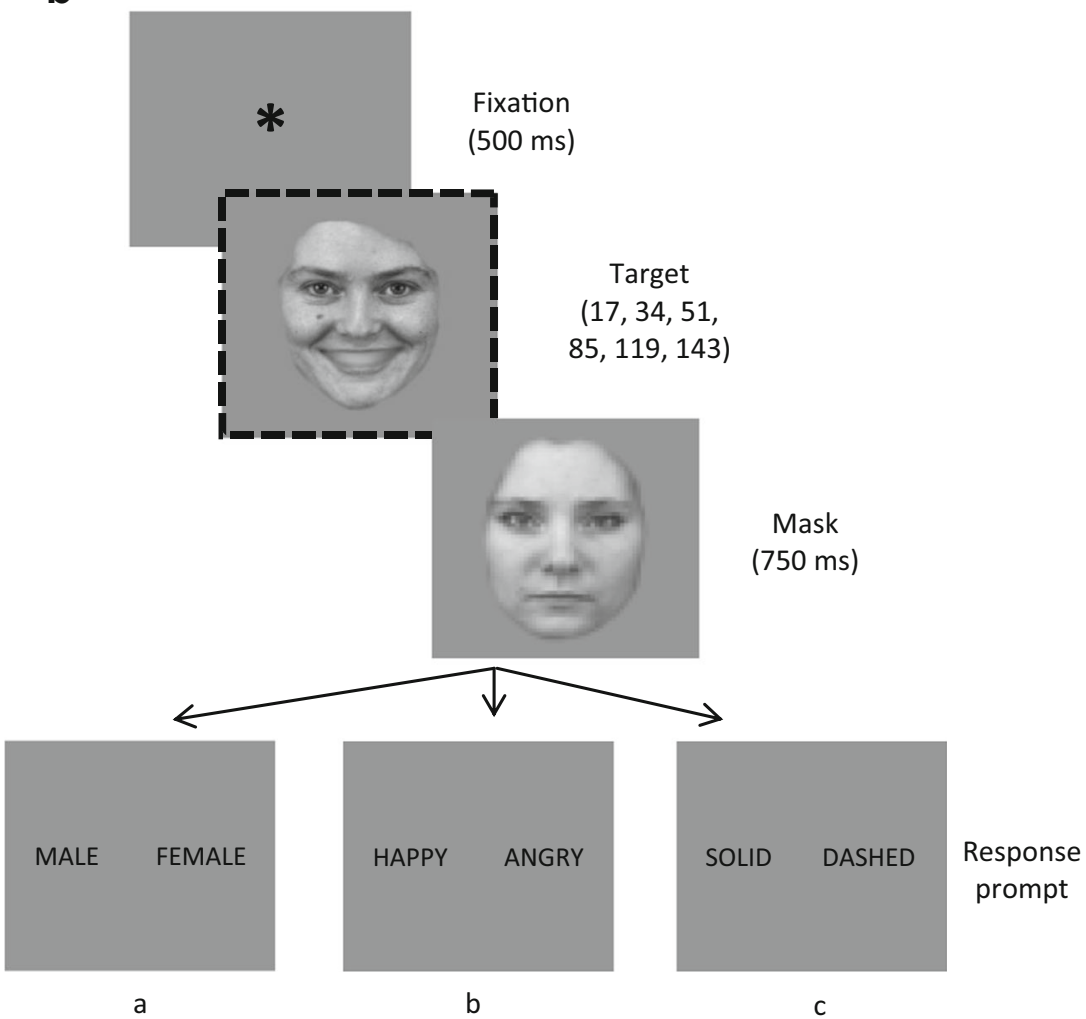

Fig. 1 (a) Samples of both male and female and angry and happy stimuli. (b) Example of trial events in the dual tasks. In the dualfacial condition the participant was asked unpredictably to identify the gender (a) or the expression (b) of the face. In the dual-control

variances (all $p>.05$, except for dual control vs. dual facial in the gender task at 143 -ms duration, $F(1,22)=7.001, p=.015)$. A normality test showed that data distributions were normally distributed, except for the expression task in the control context at $143 \mathrm{~ms}(Z=1.365, p=.048)$.

As can be seen in Fig. 2, expression was categorized with similar accuracy in the dual-facial and the expression-control conditions. On the other hand, gender categorization was clearly less accurate in the dual-facial condition. It is also interesting conditions the two possible tasks were: Gender discrimination (a) and line discrimination (c) in the gender-control condition and expression discrimination (b) and line discrimination (c) in the expressioncontrol condition

that similar accuracy of gender and expression categorization was observed in the control conditions, thus showing that the two tasks were equal in terms of difficulty in the context of the line-discrimination task. These impressions were confirmed by statistical analysis.

A $2 \times 6 \times 2$ ANOVA with Face Task (expression or gender) and Duration (17, 34, 51, 85, 119, $143 \mathrm{~ms})$ as repeated measures factors and Task Context (dual-facial and dual-control) as the between-subjects factor yielded significant main effects 


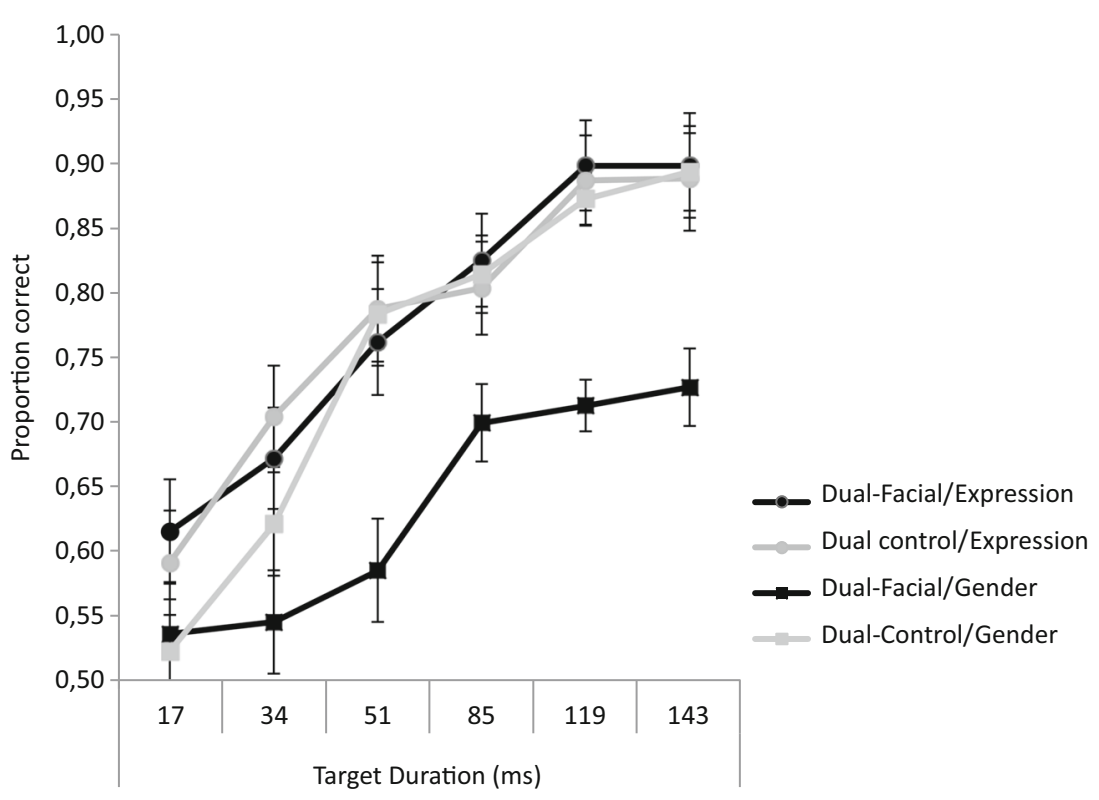

Fig. 2 Mean proportion of correct responses for the gender and expression tasks in the dual-facial and dual-control conditions in Experiment 1. Vertical bars represent standard error of the mean

for Face Task, $F(1,22)=16.08, p=.001, \eta_{\mathrm{p}}{ }^{2}=.42$, in the sense that the expression task showed higher accuracy rates than the gender task $(M=.78, S E M=.021$ and $M=.69$, $S E M=.016$ respectively). A main effect of Duration, $F(5,110)=63.31, p=.001, \eta_{\mathrm{p}}{ }^{2}=.74$ was also found, all durations differing from each other $\left(p_{s}<.05\right)$, except for the pairs 17 and $34 \mathrm{~ms}, 51$ and $85 \mathrm{~ms}$, and 119 and $143 \mathrm{~ms}$, which were non-statistically different from each other. A marginal Task Context effect was also found, $F(1,22)=3.38$, $p=.079, \eta_{\mathrm{p}}{ }^{2}=.13$ showing the dual-facial to be less accurate $(M=.71, S E M=.022)$ than the dual-control $(M=.76, S E M=$ .022). Most important, the interaction Face Task $\times$ Task Context was statistically significant, $F(1,22)=7.83$, $p=.001, \eta_{\mathrm{p}}{ }^{2}=.26$. Duration and Task Context was also significant, $F(5,110)=2.30, p=.049, \eta_{\mathrm{p}}{ }^{2}=.095$. In order to explore the interactions, post hoc analyses with Bonferroni correction were carried out, showing significant differences for Expression $(M=.79, S E M=.03)$ and Gender $(M=.63$, $S E M=.023)$ in the dual-facial task, $p=.001$, but not for Expression $(M=.77, S E M=.03)$ and Gender $(M=.75$, $S E M=.023)$ in the dual-non-facial task, $p=.40$. Moreover, the same type of comparisons between Duration and Task Context showed significant differences in all the durations $\left(p_{s}<.001\right)$ except for the fourth one $(85 \mathrm{~ms}) p=.14$. These results indicated that while expression task remained constant between task context, gender categorization was significantly impaired by the concurrent demand to attend to an additional facial feature, and this occurred from the very first duration. In other words, gender and expression were categorized with similar accuracy when the concurrent component of the dual task was the discrimination of a non-facial feature.

\section{Discussion}

Comparison of the accuracy of expression and gender discrimination under different dual-task conditions revealed a pattern of asymmetric interference between tasks. Whereas gender discrimination was significantly impaired by the concurrent demand to attend to expression, participants performed the expression task with similar accuracy when the concurrent component of the dual task was gender or line discrimination. These results show that expression and gender discrimination cannot be concurrently performed without a cost in accuracy at least under conditions of limited access to the visual input such as those employed in the present study. The fact that this cost was circumscribed to the gender task indicates that this interference is asymmetrical and suggests a priority of expression over gender identification.

A second relevant aspect of the results of Experiment 1 has to do with the effects of target duration. Two relevant results were obtained in this respect. First, a reliable trend for increased accuracy at longer target durations was observed for both gender and expression categorization. Comparisons between performances obtained at different durations suggest that at least under the specific conditions of our study a critical transition occurs within the 51- to $85-\mathrm{ms}$ range. A second result that is worth mentioning is that in the dual facial condition superior performance in the expression task was observed even at the relatively short duration of $34 \mathrm{~ms}$. In contrast to this, performance in the gender task with this duration was still at chance level. Consistent with previous results obtained in our laboratory (Aguado et al., 2014), this last result shows that gender discrimination requires additional perceptual evidence 
beyond what suffices to support basic expression analysis. However, it is important to note that no significant increases in accuracy were observed in the 85- to 143-ms range. Thus, it can be concluded that increasing the time available to gather relevant information from the target faces was not effective to reduce the asymmetric interference between the two facial tasks. Further comments on the theoretical implications of these results will be deferred until the general discussion.

One aspect that complicates the interpretation of the results obtained in this study is that we used a mixed between subjects design, with the comparison between facial tasks analyzed between subjects. This is of course not an ideal design if we want to draw firm conclusions on the differential effects of the dual facial and control conditions. Consequently, in Experiment 2 a simpler within-subjects design was used. In that design all subjects performed the dual-facial task and the gender-control and expression-control tasks on different blocks of trials.

\section{Experiment 2}

In this experiment, dual-task procedures similar to those used in Experiment 1 were employed. All subjects performed the dual-facial task and the corresponding dual-control tasks in a between-blocks design. This design allows a more appropriate comparison of discrimination performance under the different dual-task conditions. The parameters were similar to those in Experiment 1, with the exception that the target faces were presented with a constant 119-ms duration.

\section{Participants and apparatus}

Twelve undergraduate female Psychology students from the Universidad Complutense (Madrid, Spain) participated ( $M$ age $=20$, range $=19-21$ years). Apparatus and experimental conditions were similar to those of the Experiment lexcept as described below.

\section{Procedure}

Trials were distributed in three blocks. On each block the participants had to perform one of three dual tasks: expression-gender (dual-facial task), expression-line frame (dual expression-control task), and gender-line frame (gender-control task). Block order was counterbalanced between subjects. Before starting each block and with the assistance of the experimenter, 16 practice trials in the corresponding task were given. Different pairs of keys were assigned to the gender, expression, and line frame discriminations. The specific key assignment was counterbalanced between participants. Each block comprised 96 trials, 48 with each dual-task condition. The total number of trials per participant was 288 .
Based on the results of Experiment 1, one single target duration of $119 \mathrm{~ms}$ was selected to ensure that both facial tasks could be performed at their best. The aim of the present study was to compare performance under different dual-task conditions with a more appropriate, within-subjects design, and we were not interested in exploring further the effects of target duration that were well established in Experiment 1.

\section{Results and discussion}

As is shown in Fig. 3, expression discrimination was performed with similar accuracy in the experimental and control conditions. In contrast to this, poorer performance in the dual facial condition can be seen in the case of the gender task. It is important to note that the sample data met assumptions for ANOVA in this experiment (all $p>.05$ ). These impressions were confirmed by statistical analysis. A $2 \times 2$ repeated measures ANOVA, with Task Context (dual-facial or dual-control) and Facial Task (gender or expression) as the within-subjects factors showed a main effect of Task Context, $F(1,11)=20.89, p=.001, \eta_{\mathrm{p}}^{2}=.65$, having the dual-control higher accuracy rates than the dual-facial task $(M=.89, S E M=.012$ and $M=.82, S E M=.011$, respectively). Facial Task was also found to be significant, $F(1,11)=$ $22.45, p=.001, \eta_{\mathrm{p}}{ }^{2}=.67$, with higher accuracy for the expression than for the gender task $(M=.91, S E M=.016$ and $M=.81, S E M=.011$, respectively). Finally, the interaction $F(1,11)=46.47, p<.001, \eta_{\mathrm{p}}^{2}=.8$ was also found significant. Post hoc comparisons with Bonferroni correction revealed significant differences between facial tasks only in the dual-facial condition. In this condition, significantly lower accuracy was observed in the gender than in the expression task $(M=.73, S E M=.012$ and $M=.92$, SEM .02 , respectively), $p<.001$. However, the two facial tasks were performed with similar accuracy in the dual-control conditions $(M=.89$, SEM $=.019$ and $M=.89, S E M=.015$ for expression and gender, respectively). Moreover, while the expression task was performed with similar accuracy in both task contexts, gender discrimination was significantly less accurate in the dual-facial condition.

Finally, in order to confirm if the poorer gender performance under the dual facial condition was manifested with all combinations of gender and expression, a $2 \times 2 \times 2$ threeway repeated measures ANOVA was performed on accuracy data, with Task Context, Emotion, and Gender as factors. This analysis gave a significant three-way interaction, $F(1$, 11) $=108, p<.001, \eta_{\mathrm{p}}{ }^{2}=.9$. Post-hoc comparisons showed that there was a significant increase in accuracy in the control condition except in the case of happy female faces that were recognized as females with similar accuracy in both conditions. Discrimination accuracy of female faces in the dual-facial task was $M=.40(\mathrm{SEM}=.04)$ and $M=.87$ (SEM 


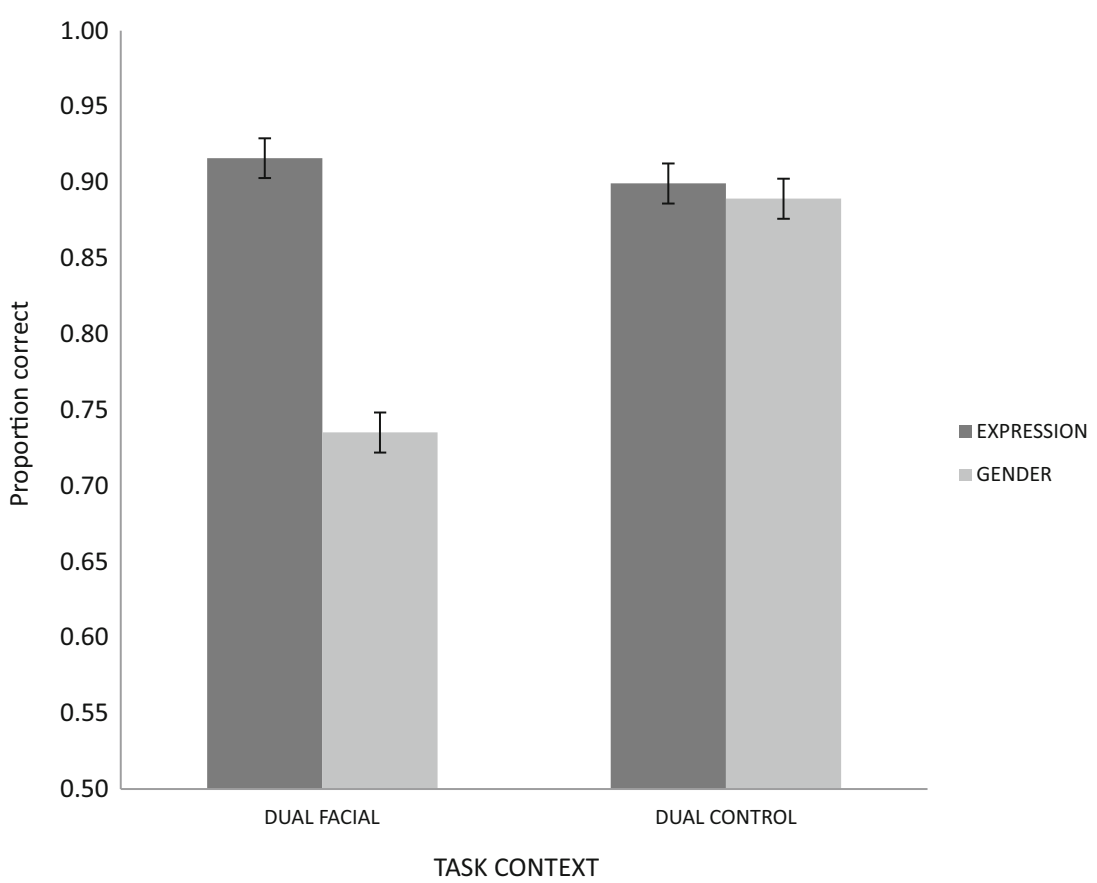

Fig. 3 Mean proportion of correct responses for the gender and expression tasks in the dual-facial and dual-control conditions in Experiment 2. Vertical bars represent standard error of the mean

$=.024)$ for angry and happy faces, respectively. The corresponding results for male faces were $M=.88$ (SEM =.026) and $M=.79(\mathrm{SEM}=.037)$. In the control condition, the accuracy of identification of female faces was $M=.92$ $(\mathrm{SEM}=.022)$ and $M=.79(\mathrm{SEM}=.024)$ for angry and happy faces, respectively. For male faces, the corresponding results were $M=.92(\mathrm{SEM}=.026)$ and $M=.91(\mathrm{SEM}=$ $.028)$.

The results of Experiment 2 in general confirmed the basic finding of Experiment 1, that is, an asymmetric interference between gender and expression in the dualfacial condition. In both experiments, poorer discrimination performance in the dual-facial than in the dual-control condition was observed only in the gender task. The fact that in the present experiment the comparison between the dual-facial and dual-control conditions was performed in a within-subject design provides more convincing support to that conclusion. The gender results presented in this experiment go hand in hand with previous results of higher accuracy for happy than for angry female faces and for angry than for happy male faces, but only in the context of a gender categorization task (see Becker et al. 2011; Hess et al., 2009; Pérez-Moreno et al., 2016). This result can be explained by the fact that angry females might be misidentified as males and happy males as females as has been reported previously in our laboratory (see Aguado, García-Gutiérrez, \& Serrano-Pedraza, 2009; Grossman \& Wood, 1993; Plant, Hyde, Keltner, \& Devine, 2000; Tay, 2015).

\section{Experiment 3}

In Experiments 1 and 2, asymmetric interference between gender and expression discrimination was observed in a task that required simultaneous attention to both facial properties. While this requirement significantly reduced the accuracy of gender discrimination, compared to the corresponding control, it had no effect on expression discrimination. However, we should mention a potential confounding factor that might have influenced the results of the dual-facial task. Male and female neutral faces were used as masks in Experiments 1 and 2. As masks were expressively neutral, there should be no specific interference between the expression of the mask and that of the immediately preceding target face. However, because gender information was present in the masks as well as in the targets, gender identification might have been interfered with in those trials in which the gender of the target and the mask was different, contributing to the poorer performance in this task. To address this problem, in the present experiment we employed as masks androgynous faces that could not be clearly perceived as masculine or feminine. In this way, the possibility of differential interference of the mask with gender discrimination or the expression of the target faces was prevented. The procedure was modeled after that of Experiment 2, the only exception being the stimuli used as masks. A replication of the main results of Experiments 1 and 2 under these new and more 
appropriate conditions would provide more convincing evidence of the asymmetric interference effect observed in those experiments.

\section{Participants and apparatus}

Seventeen psychology students from the Universidad Complutense (Madrid, Spain) participated in exchange for course credits (12 female, five male, $M$ age $=20$, range $=$ 19-27 years). Apparatus and experimental conditions were similar to those of the Experiment 1 except as described below.

\section{Procedure}

The procedure and stimuli were identical to those of Experiment 2 . The only difference was that the masks were four androgynous faces that were created based on four of the faces used as masks in Experiments 1 and 2. First, the eight faces used as masks in those experiments were morphed with the software Face Gen 3.5 using the sex morph utility of the program. Four different faces were generated from each original stimulus, corresponding to four positions along the male-female continuum. The resulting set of 32 stimuli was rated by an independent group of psychology students $(N=25)$ along a 7-point continuous scale, with anchors $1=$ very masculine, $4=$ as masculine as feminine and $7=$ very feminine. Based on these ratings, the four faces rated as most androgynous $(M=4.32, S D=.70)$ were selected to be used as masks (see Fig. 4). Similar to Experiments 1 and 2, these pictures were quantized using the average density of the original images, with square size of $8 \times 8$ pixels.

\section{Results and discussion}

Figure 5 presents the results for the four experimental conditions. It can be seen first that performance was in general more accurate in the expression than in the gender task. Crucial for the goals of this study, emotional expression was discerned with similar accuracy in the dual-facial and dual-control conditions. However, performance in the gender task was clearly

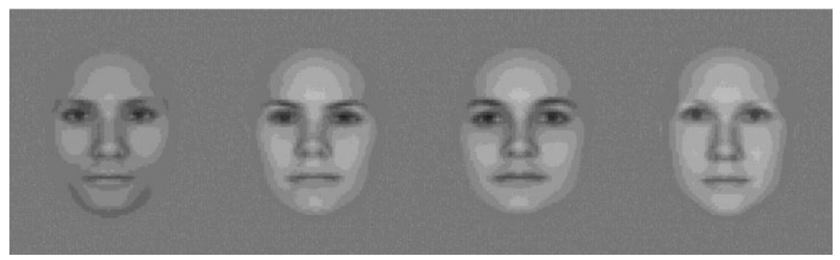

Fig. 4 Androgynous faces used as masks in Experiment 3. The stimuli are morphs of pictures AM06NES, AM11NES, AF02NES, and AF12NES of the KDEF database

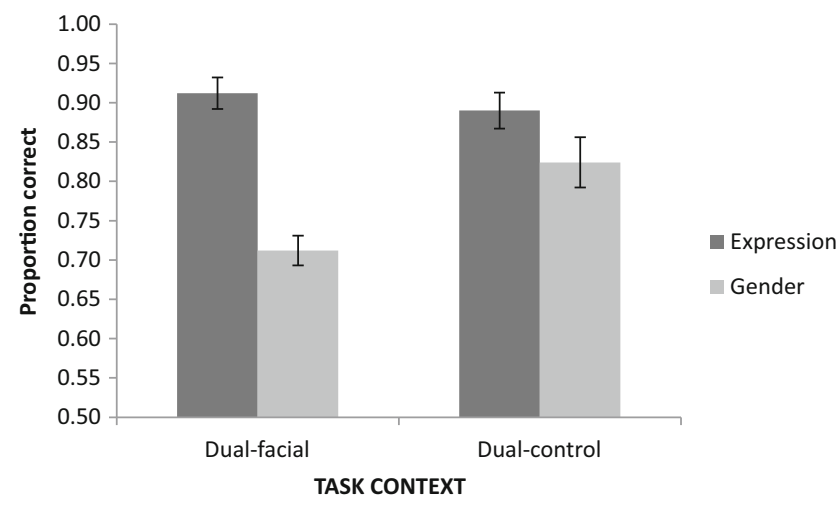

Fig. 5 Mean proportion of correct responses for the gender and expression tasks in the dual-facial and dual-control conditions in Experiment 3. Vertical bars represent standard error of the mean

inferior in the context of the dual-facial condition. These impressions were confirmed statistically. The sample data met assumptions for ANOVA in this experiment (all $p>.05$ ).

A $2 \times 2$ ANOVA, with Task Context (dual-facial or dualcontrol) and Facial Task (gender or expression) as the withinsubjects factors, showed main effects of Task Context, $F(1$, 16) $=9.07, p=.008, \eta_{\mathrm{p}}{ }^{2}=.36$, with higher accuracy rates in the dual-control compared with the dual-facial task $(M=.857$, $S E M=.026$ and $M=.812, S E M=.017$ respectively), as well as Facial Task, $F(1,16)=98, p=.001, \eta_{\mathrm{p}}^{2}=.86$, the expression task being superior to the gender task $(M=.901, S E M=$ .02 and $M=.77, S E M=.022$, respectively). Finally, this interaction was also found to be significant, $F(1,16)=16.6$, $p=.001, \eta_{\mathrm{p}}{ }^{2}=.51$. Post hoc comparisons with Bonferroni correction revealed significant differences between task contexts only in the case of the gender task $(M=.824, S E M=.032$ and $M=.712, S E M=.019$ for dual-control and dual-facial, respectively), $p=.001$.

A decrease in performance in the dual-facial task was again shown for gender but not for expression discrimination. In the present experiment this result was obtained using androgynous faces as masks. This allows us to rule out the contribution of the interference between the gender of mask and the target faces to the impaired gender discrimination in the dualfacial task.

An additional issue refers to the generality of our findings. In the present series of experiments, only happy and angry expressions were used and we do not know if the results can be generalized to other facial expressions. For example, our results might depend on the use of targets of opposite affective valence. This might have increased the attentional demands of the expression task, thus contributing to divert the resources needed for gender identification. Additionally, there are differences in the communicative function of different facial expressions that might determine their power to capture attention. For example, the smile and the frown that are characteristic of happy and angry faces, respectively, are directed at the observer. However, this is not necessarily so with expressions 
of sadness, fear or disgust, that may be produced in the absence of an external observer. It might well be that the power to capture preferential attention depends on the extent to which the observer feels that the expression is directed at her/him. To test for these possibilities in Experiments $4 \mathrm{a}$ and $4 \mathrm{~b}$ different target expressions were employed in with a design similar to that of the present experiment.

\section{Experiments $4 a$ and $4 b$}

In Experiments 1-3 a differential impact of the facial dualtask condition on gender and expression identification was observed using as targets faces showing angry and happy expressions. This finding suggests that at least under conditions of limited access to visual information, such as those employed in our studies, expression processing has priority over gender processing. However, in the former experiments the expressions used do not allow inference of whether the effect can be generalized to other facial expressions. In Experiments $4 \mathrm{a}$ and $4 \mathrm{~b}$ different combinations of emotions were employed as targets using a design similar to that of Experiment 3. In Experiment 4a two expressions of the same valence (fear and disgust) were used and in Experiment $4 \mathrm{~b}$ we used again a positive and a negative expression but replaced angry with fearful expressions.

\section{Participants, apparatus, and procedure}

Thirty-two undergraduate students from Universidad Complutense de Madrid (Madrid, Spain) participated in exchange for course credits. Eight males and 24 females composed the sample and the median $(M)$ age was 19.83 (range 18-29 years).

Apparatus and procedure were identical to those of Experiment 3, except for the nature of expressions used as targets. The new stimuli used in Experiment 4 a were 32 pictures of faces showing expressions of fear or disgust (eight male and eight female faces for each expression) selected from the KDEF collection. In Experiment $4 b$ the same set of happy faces used in Experiments 1-3 were employed, plus the same set of fearful expressions used in Experiment $4 \mathrm{a}$.

\section{Results and discussion}

The analysis of the data obtained in Experiment $4 \mathrm{a}$ did not show any significant main or interaction effects (all $p_{s}>.05$ ). In the case of Experiment $4 \mathrm{~b}$, the analysis showed only a main effect of Facial Task, $F(1,17)=12.32, p=.03, \eta_{\mathrm{p}}{ }^{2}=.42$, with poorer performance in the gender than in the expression task $(M=.79, \mathrm{SEM}=.045$ and $M=.94, \mathrm{SEM}=.011$, respectively $)$.
Neither the main effect of Task Context nor the interaction was found to be significant (all $p_{s}>.05$ ).

The results reported in the former experiments were not replicated in Experiments $4 \mathrm{a}$ and $4 \mathrm{~b}$ using pairs of expression targets that were different to those employed in Experiments 1-3. More specifically, when two negative expressions, fear and disgust (Experiment 4a), or when happy and angry faces were used as targets (Experiment $4 \mathrm{~b}$ ), the effect of impaired gender discrimination in the dual-facial task condition disappeared. Thus, in the absence of further evidence with other facial expressions we must limit the conclusions based on the results of Experiments 1-3 to the particular case of the discrimination between happy and angry expressions.

\section{General discussion}

The efficiency of gender and expression discrimination was studied using a dual-task paradigm with backward masked faces. A different pattern of results was obtained depending on the specific combination of target emotional expressions. In Experiments 1-3 a pattern of asymmetric interference in the discrimination of facial gender and expression under dual-task conditions was observed. The demand to attend simultaneously to these facial properties (dualfacial task context) impaired gender performance but left expression performance unchanged compared to the appropriate control conditions. This result was obtained in Experiment 1 in a design where the dual facial and control conditions were compared between subjects. In this experiment the effect of different target durations was also studied. Impaired gender discrimination in the dual-facial task was observed over the 51- to 143-ms range of target durations that corresponds to the time range where gender was differentiated above chance level. It is important to note that asymptotic performance in the gender task was observed at the 85-ms target duration, showing that allowing more time to extract relevant information from the face did not resulted in improved discrimination. Our interpretation is that the demands of the dual task imposed limits to the use of facial information and that these limits were set by the superior attention-grabbing power of the facial features that are relevant for expression discrimination. In relation to the more general issue of the independence between gender and expression processing, our results suggest that processing of these facial properties is limited under conditions that tax attentional resources. Restricted exposure to the stimulus produced by short exposure times and backward masking as in the present experiments seems to give more weight to expression-relevant features, preventing or making the processing more difficult. 
Experiment 2 replicated the main finding of impaired gender discrimination in the dual-facial condition in a within-subjects design with one single target duration. Therefore, in Experiment 3 androgynous faces were used as masks to exclude the possibility that in the previous experiments the gender of the face masks might have specifically interfered with processing of the gender of the target faces. In spite of this variation, the same pattern of impaired gender discrimination but preserved expression in the dual facial context was still found. Thus, these three experiments coincide in showing that gender and expression discrimination cannot be performed concurrently without a cost in accuracy, and that this cost is asymmetrical and affects only gender discrimination. This was observed under conditions in which access to visual input was restricted to a brief period of time and that required that information was gathered in a single visual fixation. Under these conditions, the dual-facial task imposes stringent demands on processing resources and attention is preferentially captured by highly salient expression features, resulting in less accurate gender discrimination.

The contrast between the effects of dual-task demands on gender and expression discrimination in Experiments 1-3 can be attributed to differences in the way that these facial properties are processed and to the higher salience of the features that have diagnostic value for the recognition of emotional expression. More specifically, our interpretation is that under the restricted viewing conditions of the present study, attention was captured by salient expression features to the detriment of attention to gender-relevant features. Consistent with this interpretation, previous research has shown that fast and efficient identification of angry and happy expressions may occur based on characteristic single features. The upturned lip corners characteristic of the smile is the main facial marker of the facial expression of happiness and it appears to be sufficient to identify happy expressions (e.g., Calvo \& Nummenmäa, 2008; Smith, Cottrell, Gosselin, \& Schyns, 2005). On the other hand, the eyebrow frown is the more reliable and salient marker of angry expressions (e.g., Bombari et al., 2013; Fox \& Damjanovic, 2006; Smith et al., 2005), although changes in the mouth region (tight closed lips or tense open mouth) can also provide efficient recognition (Horstmann \& Bauland, 2006). This is not to deny the important role that configural information derived from relations between individual features or from holistic processing of the face has for expression recognition (e.g., Bombari et al., 2013; Calder, Young, Keane, \& Dean, 2000; Durand et al., 2007). Moreover, recognition of different expressions may rely to a different extent on featural and configural information, and this may also depend on the intensity with which the expression is shown. What we maintain is that at least in the case of happy and angry faces and under the restricted viewing conditions we employed, emotional expression can be recognized based on single salient features. In contrast to the crucial role of these features in expression discrimination, a wider set of twodimensional, three-dimensional, and texture cues have diagnostic value for gender discrimination (e.g., Bruce et al., 1993; Bruce \& Langton, 1994; Yamaguchi, Hirukawa, \& Kanazawa, 1995). Thus, it can be assumed that because more perceptual evidence is required, more detailed processing of faces is needed for gender discrimination. Under the restricted viewing conditions used in the present experiment, attention in the dual-facial task would have been captured by the salient expression features, interfering with the slower and more detailed processing needed for gender discrimination.

One way in which attention to emotional expression in Experiments 1-3 might have interfered with accurate gender discrimination in the dual-facial task is by biasing gender recognition in a direction that is consistent with expression features. Previous studies on the interaction between the gender and expression of faces have shown that gender recognition is influenced by emotional expression. While angry expressions make faces look more masculine and reduce the accuracy with which a face is recognized as female (Aguado et al., 2009; Hess, Adams, Grammer, \& Kleck, 2009), the smile makes faces look less masculine and impairs recognition of a face as male (Hess et al., 2009; Kawamura, Komori, \& Miyamoto, 2008). The explanation of these effects is that some facial features have common diagnostic value for gender and expression. For example, because a reduced brow-to-lid distance is a feature common to male and to angry faces (e.g., Burton, Bruce, \& Dench, 1993; Ekman \& Friesen, 1978), a female face showing an angry expression may be misidentified as male. In the absence of additional information that might be supported by other gender-relevant features (because of occluded view or brief exposure time, for example), misidentifications would be especially likely. If in our dual-facial task attention was captured preferentially by the more salient expression features, that is, the smiling mouth of happy faces and the eyebrow frown of angry ones, errors in gender identification would be expected. Furthermore, the brief exposure times would have impeded more detailed visual inspection and analysis of other genderrelevant features that might counteract the influence of the salient expression features. According to this account, gender misidentifications should have been more likely in the cases of angry female and happy male faces (Aguado et al., 2009; Grossman \& Wood, 1993; Tay, 2015). In fact, the accuracy of gender discrimination in Experiment 2 for the different combinations of gender and expression confirms this prediction is a better discrimination of gender for happy male than for angry female faces. It seems that the demands of the dualfacial task led to increased gender misidentifications precisely for those faces showing expressions that bias gender 
recognition in the wrong direction, that is, angry for female faces and happy for male faces. Even more, these were the same faces for which a superior increase in accuracy was shown in the absence of the demand to simultaneously attend to emotional expression.

Another factor that must be considered in order to explain the asymmetry interference effect observed in Experiments 1-3 has to do with the role of task instructions in modulating attention and stimulus processing. The same set of happy and angry faces was used in the dual facial and control conditions, so the faces had the same salient expression features under both conditions. However, these features had a disproportionate effect in the dual-facial task. If the interpretation offered for the results presented in this study is correct, it has to be assumed that a task context that explicitly requires simultaneous attention to both gender and expression acts by selectively biasing attention to the more salient, expression-relevant cues. If this is so, we should then conclude that when the participant is given the assignment of identifying both the gender and the expression of the faces, primacy is automatically given to expression processing and attention is selectively focused on expression-relevant features. In other words, the ability of task demands to modulate perceptual processing and the assignment of attentional resources would depend on the specific content of those demands. At this point, we can only speculate that the relative primacy of expression suggested by the present results is related to the high relevance of facial expressions of emotion as social signals and to the need to prioritize the use of processing resources under conditions of minimal exposure to visual input.

The interference of gender discrimination in the dualfacial task shown in Experiments 1-3 with angry and happy targets was not observed in Experiments $4 \mathrm{a}$ and $4 \mathrm{~b}$ with different target combinations (fear and disgust expressions in Experiment $4 \mathrm{a}$ and fear and happy expressions in Experiment 4b). Under these new conditions gender was differentiated with similar efficiency under the dual facial and control conditions. A tentative explanation for this discrepancy is in terms of the communicative functions of different facial expressions and the superior power of angry and happy expressions to command attention and engage preferential processing that, as was mentioned in the introduction, has been shown in visual search studies.

Facial expressions convey valuable social information that differs in terms of their expressive and communicative functions (Ekman, 1997). From an evolutionary point of view, facial expressions of emotion convey valuable social information but the precise expressive and communicative functions differ between expressions. From this perspective a characteristic of the expressions of happiness and anger is that they are directly addressed at the perceiver. While the basic communicative function of angry expressions is to communicate threat, that of happy expressions is to signal a positive disposition to approach or to be approached. In both cases, the target to which the expression is addressed is the perceiver. What we propose is that this functional characteristic increases the power of angry and happy expressions to command attention at the expense of other facial features such as those relevant for gender discrimination. This hypothesis suggests a fast and accurate detection of angry and happy faces (Eastwood, Smilek, \& Merikle., 2001; Reynolds, Eastwood, Partanen, Frischen, $\&$ Smilek, 2009; Tay, 2015). The ability of angry and happy faces to command attention would be reinforced when the task assigned to the participant is precisely to differentiate between them. This would be so because the information provided by happy and angry expressions requires clearly differentiated responses from the perceiver, namely positive and negative approaches (e.g., Harmon-Jones, Schmeichel, Mennitt, \& Harmon-Jones, 2011). This would not be so when the participant is asked to differentiate between two negative expressions such as anger and fear or between happy and fearful faces. Consistent with this approach, it has been shown that whereas direct gaze facilitates processing of the approach-oriented emotions of anger and joy, averted gaze facilitates processing of avoidance-oriented emotions such as fear and sadness (Adams \& Kleck, 2003). Given that all the expressions used as targets in our experiments showed gaze directed at the perceiver, we assume that processing of happy and angry expressions was selectively facilitated and that this was especially so when the participant had to differentiate between them. Of course, this explanation of the discrepancy between the results of Experiments 1-3 and those of Experiments $4 \mathrm{a}$ and $4 \mathrm{~b}$ is tentative, and more firm conclusions must await evidence from new studies. This evidence should come from studies testing different combinations of expression targets. Moreover, relevant evidence for our explanation might come from studies testing the effects of the interaction between the expression shown by the face and the direction of gaze, comparing faces with direct and averted gaze (for a review see Rigatto \& Farroni, 2003).

To sum up, the results reported in the present series of experiments suggest a complex pattern of interactions between the gender and emotional expression of faces under conditions of restricted exposure that tax the attentional and perceptual resources of the participant. It seems that the precise pattern of interaction and the ability to attend simultaneously to the features relevant for the discrimination of these facial properties depend on the specific combination of the expressions used as targets. More specifically, we suggest that a crucial factor is the ability of different expressions to command preferential attention, based on their different expressive and communicative functions. 
Author note This work was supported by grant PSI2013-44262-P to Luis Aguado from the Spanish Ministerio de Economía y Competitividad. Participation of Verónica Romero-Ferreiro was made possible by a Predoctoral fellowship from the Complutense University of Madrid, Spain. We thank Dr. Ignacio Serrano-Pedraza for his help with image processing.

\section{References}

Adams, R. B., \& Kleck, R. E. (2003). Perceived gaze direction and the processing of facial displays of emotion. Psychological Science, 14(6), 644-647.

Aguado, L., García-Gutiérrez, A., \& Serrano-Pedraza, I. (2009). Symmetrical interaction of sex and expression in face classification tasks. Attention, Perception, \& Psychophysics, 71(1), 9-25.

Aguado, L., Serrano-Pedraza, I., \& García-Gutiérrez, A. (2014). A comparison of backward masking of faces in expression and gender identification tasks. Psicológica, 35, 171-194.

Atkinson, A. P., Tipples, J., Burt, D. M., \& Young, A. W. (2005). Asymmetric interference between sex and emotion in face perception. Perception \& Psychophysics, 67, 1199-1213.

Batty, M., \& Taylor, M. J. (2003). Early processing of the six basic facial emotional expressions. Cognitive Brain Research, 17(3), 613-620.

Becker, D. V., Anderson, U. S., Mortensen, C. R., Neufeld, S. L., \& Neel, R. (2011). The face in the crowd effect unconfounded: Happy faces, not angry faces, are more efficiently detected in single- and multipletarget visual search tasks. Journal of Experimental Psychology: General, 140(4), 637-659.

Bombari, D., Schmid, P. C., Schmid Mast, M., Birri, S., Mast, F. W., \& Lobmaier, J. S. (2013). Emotion recognition: The role of featural and configural face information. The Quarterly Journal of Experimental Psychology, 66(12), 2426-2442.

Breitmeyer, B. G., \& Ogmen, H. (2000). Recent models and findings in visual backward masking: a comparison, review, and update. Perception \& Psychophysics, 62,1572-1595.

Bruce, V., Burton, M. A., Hanna, E., Healey, P., Mason, O., Coombes, A., \& Linney, A. (1993). Sex discrimination: How do we tell the difference between male and female faces? Perception, 22,131-152.

Bruce, V., \& Langton, S. (1994). The use of pigmentation and shading information in recognising the sex and identities of faces. Perception, 23,803-822.

Bruce, V., \& Young, A. (1986). Understanding face recognition. British Journal of Psychology, 77,305-327.

Burton, A. M., Bruce, V., \& Dench, N. (1993). What's the difference between men and women: Evidence from facial measurement. Perception, 22,153-176.

Calder, A. J., Young, A. W., Keane, J., \& Dean, M. (2000). Configural information in facial expression perception. Journal of Experimental Psychology: Human Perception and Performance, 26(2), 527.

Calvo, M. G., \& Lundqvist, D. (2008). Facial expressions of emotion (KDEF): Identification under different display-durationconditions. Behavior Research Methods, 40(1), 109-115.

Calvo, M. G., \& Nummenmaa, L. (2008). Detection of emotional faces: salient physical features guide effective visual search. Journal of Experimental Psychology: General, 137,471-494.

Calvo, M., \& Esteves, F. (2005). Detection of emotional faces: Low perceptual threshold and wide attentional span. Visual Cognition, 12(1), 13-27.

Compton, R. J. (2003). The interface between emotion and attention: a review of evidence from psychology and neuroscience. Behavioral and Cognitive Neuroscience Reviews, 2(2), 115-129.

Craig, B. M., Becker, S. I., \& Lipp, O. V. (2014). Different faces in the crowd: a happiness superiority effect for schematic faces in heterogeneous backgrounds. Emotion, 14,794-803. doi:10.1037 /a0036043

Desimone, R., \& Duncan, J. (1995). Neural mechanisms of selective visual attention. Annual Review of Neuroscience, 18(1), 193-222.

Durand, K., Gallay, M., Seigneuric, A., Robichon, F., \& Baudouin, J. Y. (2007). The development of facial emotion recognition: The role of configural information. Journal of Experimental Child Psychology, 97(1), 14-27.

Dzhelyova, M. P., Ellison, A., \& Atkinson, A. P. (2011). Eventrelatedrepetitive TMS reveals distinct, critical roles for right OFA and bilateral posterior STS in judging the sex and trustworthiness of faces. Journal of Cognitive Neuroscience, 23(10), 2782-2796.

Eastwood, J. D., Smilek, D., \& Merikle, P. M. (2001). Differential attentional guidance by unattended faces expressing positive and negative emotion. Attention, Perception \& Psychophysics, 63(6), 1004 1013.

Ekman, P. (1997). Should we call it expression or communication? Innovation: The European Journal of Social Science Research, 10(4), 333-344.

Ekman, P., \& Friesen, W. V. (1978). Facial action coding system: A technique for the measurement of facial movement. Palo Alto, CA: Consulting Psychologists Press.

Esteves, F., \& Öhman, A. (1993). Masking the face: Recognition of emotional facial expressions as a function of the parameters of backward masking. Scandinavian Journal of Psychology, 34,1-18.

Fox, E., \& Damjanovic, L. (2006). The eyes are sufficient to produce a threat superiority effect. Emotion, 6(3), 534.

Frischen, A., Eastwood, J. D., \& Smilek, D. (2008). Visual search for faces with emotional expressions. Psychological Bulletin, 134(5), 662.

Grossman, M., \& Wood, W. (1993). Sex differences in intensity of emotional experience: a social role interpretation. Journal of Personality and Social Psychology, 65(5), 1010. doi:10.1037/00223514.65.5.1010

Haberman, J., \& Whitney, D. (2007). Rapid extraction of mean emotion and gender from sets of faces. Current Biology, 17(17), R751-R753.

Harmon-Jones, C., Schmeichel, B. J., Mennitt, E., \& Harmon-Jones, E. (2011). The expression of determination: similarities between anger and approach-relatedpositive affect. Journal of Personality and Social Psychology, 100(1), 172.

Haxby, J. V., Hoffman, E. A., \& Gobbini, M. I. (2000). The distributed human neural system for face perception. Trends in Cognitive Sciences, 4,223-233.

Hess, U., Adams, R. B., Grammer, K., \& Kleck, R. E. (2009). Face gender and emotion expression: Are angry women more like men? Journal of Vision, 9(12), 19.

Horstmann, G., \& Bauland, A. (2006). Search asymmetries with real faces: testing the anger-superiorityeffect. Emotion, 6(2), 193.

Itti, L., \& Koch, C. (2001). Computational modelling of visual attention. Nature Reviews Neuroscience, 2(3), 194-203.

Juth, P., Lundqvist, D., Karlsson, A., \& Öhman, A. (2005). Looking for foes and friends: Perceptual and emotional factors when finding a face in the crowd. Emotion, 5,379-395.

Kawamura, S., Komori, M., \& Miyamoto, Y. (2008). Smiling reduces masculinity: Principal component analysis applied to facial images. Perception, 37(11), 1637-1648.

Leppänen, J. M., \& Hietanen, J. K. (2004). Positive facial expressions are recognized faster than negative facial expressions, but why? Psychological Research, 69,22-29.

Lundqvist, D., Flykt, A., \& Öhman, A. (1998). The Karolinska directed emotional faces-KDEF[CD-ROM]. Stockholm: Karolinska Institutet, Department of Clinical Neuroscience, Psychology section.

Marois, R., \& Ivanoff, J. (2005). Capacity limits of information processing in the brain. Trends in Cognitive Sciences, 9,296-305. 
Maxwell, J., \& Davidson, R. (2004). Unequally masked: Indexing differences in the perceptual salience of" unseen" facial expressions. Cognition and Emotion, 18(8), 1009-1026.

Milders, M., Sahraie, A., \& Logan, S. (2008). Minimum presentation time for masked facial expression discrimination. Cognition and Emotion, 22(1), 63-82.

Mogg, K., \& Bradley, B. P. (1999). Orienting of attention to threatening facial expressions presented under conditions of restricted awareness. Cognition and Emotion, 13,713-740.

Morris, J. S., Öhman, A., \& Dolan, R. J. (1998). Conscious and unconscious emotional learning in the human amygdale. Nature, 393,467470.

Mouchetant-Rostaing, Y., Giard, M. H., Bentin, S., Aguera, P. E., \& Pernier, J. (2000). Neurophysiological correlates of face gender processing in humans. European Journal of Neuroscience, 12(1), 303310 .

Mouchetant-Rostaing, Y., \& Giard, M. H. (2003). Electrophysiological correlates of age and gender perception on human faces. Journal of Cognitive Neuroscience, 15(6), 900-910.

Öhman, A., Lundqvist, D., \& Esteves, F. (2001). The face in the crowd revisited: a threat advantage with schematic stimuli. Journal of Personality and Social Psychology, 80,381-396.

Pérez-Moreno, E., Romero-Ferreiro, V., \& García-Gutiérrez, A. (2016). Where to look when looking at faces: visual scanning is determined by gender, expression and tasks demands. Psicológica, 37,127-150.

Pessoa, L., Jappee, S., \& Ungerleider, L. G. (2005). Visual awareness and the detection of fearful faces. Emotion, 5,243-247.

Pinkham, A. E., Griffin, M., Baron, R., Sasson, N. J., \& Gur, R. C. (2010). The face in the crowd effect: anger superiority when using real faces and multiple identities. Emotion, 10,141.

Plant, E. A., Hyde, J. S., Keltner, D., \& Devine, P. G. (2000). The gender stereotyping of emotions. Psychology of Women Quarterly, 24(1), 81-92. doi:10.1111/j.1471-6402.2000.tb01024.x

Reddy, L., Wilken, P., \& Koch, C. (2004). Face-genderdiscrimination is possible in the near-absenceof discrimination. Journal of Vision, 4,106-117.

Reynolds, M. G., Eastwood, J. D., Partanen, M., Frischen, A., \& Smilek, D. (2009). Monitoring eye movements while searching for affective faces. Visual Cognition, 17(3), 318-333. doi:10.1080 $/ 13506280701623704$

Roesch, E., Sander, D., Mumenthaler, C., Kerzel, D., \& Scherer, K. (2010). Psychophysics of emotion: The QUEST for Emotional Attention. Journal of Vision, 10(3), 4-1-9.

Rigatto, S., \& Farroni, T. (2003). The Role of Gaze in the Processing of Emotional Facial Expressions. Emotion Review, 5(1), 36-40.

Schupp, H. T., Flaisch, T., Stockburger, J., \& Junghöfer, M. (2006). Emotion and attention: event-relatedbrain potential studies. Progress in Brain Research, 156,31-51.

Schweinberger, S. R., \& Soukup, G. R. (1998). Asymmetric relationships among perceptions of facial identity, emotion, and facial speech. Journal of Experimental Psychology: Human Perception and Performance, 24,1748-1765.

Schyns, P., \& Oliva, A. (1999). Dr. Angry and Mr. Smile: when categorization flexibly modifies the perception of faces in rapid visual presentations. Cognition, 69,243-265.

Shasteen, J. R., Sasson, N. J., \& Pinkham, A. E. (2014). Eye Tracking the Face in the Crowd Task: Why Are Angry Faces Found More Quickly? PLoS ONE, 9(4), e93914. doi:10.1371/journal. pone.0093914

Smith, M. L., Cottrell, G. W., Gosselin, F., \& Schyns, P. G. (2005). Transmitting and decoding facial expressions. Psychological Science, 16(3), 184-189.

Tay, P. K. C. (2015). The adaptive value associated with expressing and perceiving angry-maleand happy-femalefaces. Frontiers in Psychology, 6,851.

Valdés-Conroy, B., Aguado, L., Fernández-Cahill, M., Romero-Ferreiro, V., \& Diéguez-Risco, T. (2014). Following the time course of face gender and expression processing: A task-dependentERP study. International Journal of Psychophysiology, 92(2), 59-66.

Vuilleumier, P. (2005). How brains beware: Neural mechanisms of emotional attention. Trends in Cognitive Sciences, 9,585-594.

Vuilleumier, P., Armony, J. L., Driver, J., \& Dolan, R. J. (2001). Effects of attention and emotion on face processing in the human brain: An event-relatedfMRI study. Neuron, 30,1-20.

Yamaguchi, M., Hirukawa, T., \& Kanazawa, S. (1995). Judgment of gender through facial parts. Perception, 24,563-575. 